\title{
Phylogenetic analysis of a gene cluster encoding an additional, rhizobial-like type III secretion system that is narrowly distributed among Pseudomonas syringae strains
}

Anastasia D Gazi ${ }^{1,2,3}$, Panagiotis F Sarris ${ }^{1,4}$, Vasiliki E Fadouloglou ${ }^{1,5}$, Spyridoula N Charova ${ }^{1}$, Nikolaos Mathioudakis ${ }^{1}$ Nicholas J Panopoulos ${ }^{1+}$ and Michael Kokkinidis ${ }^{1,2^{*}}$

\begin{abstract}
Background: The central role of Type III secretion systems (T3SS) in bacteria-plant interactions is well established, yet unexpected findings are being uncovered through bacterial genome sequencing. Some Pseudomonas syringae strains possess an uncharacterized cluster of genes encoding putative components of a second T3SS (T3SS-2) in addition to the well characterized Hrcl T3SS which is associated with disease lesions in host plants and with the triggering of hypersensitive response in non-host plants. The aim of this study is to perform an in silico analysis of T3SS-2, and to compare it with other known T3SSs.

Results: Based on phylogenetic analysis and gene organization comparisons, the T3SS-2 cluster of the $P$. syringae pv. phaseolicola strain is grouped with a second T3SS found in the pNGR234b plasmid of Rhizobium sp. These additional T3SS gene clusters define a subgroup within the Rhizobium T3SS family. Although, T3SS-2 is not distributed as widely as the HrCl T3SS in P. syringae strains, it was found to be constitutively expressed in $P$. syringae pv phaseolicola through RT-PCR experiments.

Conclusions: The relatedness of the P. syringae T3SS-2 to a second T3SS from the pNGR234b plasmid of Rhizobium sp., member of subgroup II of the rhizobial T3SS family, indicates common ancestry and/or possible horizontal transfer events between these species. Functional analysis and genome sequencing of more rhizobia and P. syringae pathovars may shed light into why these bacteria maintain a second T3SS gene cluster in their genome.
\end{abstract}

Keywords: Hrc IType III secretion system, Pseudomonas syringae, Rhizobium Type III secretion system, Phylogenetic analysis, Pathogenicity, Gene organization, Horizontal transfer events, Common ancestry, Evolutionary relationships, RT-PCR

\section{Background}

Gram-negative proteobacteria deploy various types of protein secretion systems for exporting selected sets of proteins to the cell surface, the extracellular space or into host cells [1,2]. Type III Secretion Systems (T3SS) are directly related to pathogenicity or to symbiosis with higher organisms and constitute essential mediators of the interactions

\footnotetext{
* Correspondence: kokkinid@imbb.forth.gr

${ }^{\dagger}$ Equal contributors

'Department of Biology, University of Crete, Vasilika Vouton, P.O. Box 2208, Heraklion, Crete GR 71409, Greece

${ }^{2}$ Institute of Molecular Biology \& Biotechnology, Foundation of Research \&

Technology, Vasilika Vouton, Heraklion, Crete GR 71110, Greece

Full list of author information is available at the end of the article
}

between gram-negative bacterial cells and eukaryotic ones [3-8] as the T3SS efficiently translocates bacterial proteins (effectors) directly into the host cell cytoplasm when fully developed.

The T3SS apparatus comprises three distinct parts: a) the basal body, which forms a cylindrical base that penetrates the two bacterial membranes and the periplasmic space; b) the extracellular part with the needle or the pilus as its main feature which is formed through the polymerization of specialized protein subunits that are T3SS substrates themselves; and c) the cytoplasmic part, which forms the export gate for secretion control. This apparatus is built by specific core proteins encoded by a

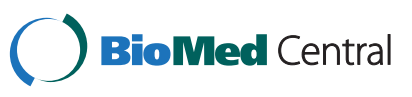


conserved subset of genes tightly organized in gene clusters with counterparts in the bacterial flagellum [6,7].

Phylogenetic analyses of the core T3SS proteins revealed that the T3S systems evolved into seven distinct families that spread between bacteria by horizontal gene transfer. (1) The Ysc-T3SS family, named after the archetypal Yersinia system, is present in $\alpha-, \beta-, \gamma^{-}$, and $\delta$ - proteobacteria. At least in $\alpha$-proteobacteria the system confers resistance to phagocytosis and triggers macrophage apoptosis. (2) The Ssa-Esc-T3SS family is named after the archetypal T3SS of enteropathogenic and enterohemorrhagic E.coli. (3) The Inv-Mxi-Spa-T3SS family named after the Inv-Spa system of Salmonella enterica and the Inv-Mxi T3S system of Shigella spp.. The family members trigger bacterial uptake by nonphagocytic cells. (4) The Hrc-Hrp1- and (5) the Hrc-Hrp2-T3SS families are present in plant pathogenic bacteria of the genus Pseudomonas, Erwinia, Ralstonia and Xanthomonas. The two families are differentiated on the basis of their genetic loci organization and regulatory systems. (6) The Rhizobiales-T3SS family (hereafter referred to as RhcT3SS) is dedicated to the intimate endosymbiosis serving nitrogen fixation in the roots of leguminous plants. (7) Finally the Chlamydiales-T3SS is present only in these strictly intracellular nonproteobacteria pathogens $[8,9]$. The phylogenetic trees obtained by the above analysis were totally incongruent with the evolutionary tree of bacteria based on $16 \mathrm{~S}$ rRNA sequences. These results imply that T3S systems did not originate within their present host bacteria, but spread through horizontal gene transfer events [9]. Furthermore, apart from a high degree of gene homologies within the T3SS families, the overall genetic organization (synteny) is also conserved [8].

In this study, we present a detailed phylogenetic and gene synteny analysis of core T3SS proteins. This analysis reveals the presence of three distinct $R h c$-T3SS family subgroups. From these subgroups, the one designated as subgroup II was found to comprise T3S systems from various Pseudomonas syringae strains as well as from Rhizobium sp. NGR234. The T3SS of subgroup II will be hereafter referred to as T3SS-2, because these systems exist in their bacterial hosts next to the wellstudied T3SS from the pNGR234a plasmid of Rhizobium sp. and the Hrc1-Hrp1 T3S system of P. syringae. Interestingly, at least two of the genes from the additional T3SS-2 gene cluster in P. syringae pv phaseolicola strain 1448 a were found to be transcriptionally active.

\section{Methods}

Sequence analysis

Genomic regions

The regions comprising and surrounding the T3SS-2 gene clusters of $P$. syringae pv phaseolicola $1448 \mathrm{a}, P$. syringae $\mathrm{pv}$ oryzae str. 1_6, P. syringae pv tabaci ATCC11528, Rhizobium spp. NGR234 and the regions comprising and surrounding the unique T3SS gene clusters of Bradyrhizobium japonicum USDA 110, Rhizobium etli CIAT 652 and R. etli CNF 42 were retrieved from the NCBI Genome database. In the cases of $P$. syringae pv tabaci ATCC11528 and $P$. syringae pv aesculi the nucleotide sequence in the region close to the T3SS gene cluster was retrieved (GenBank: N ${ }^{\circ}$ ACHU01000133 and $N^{\circ}$ ACXS0100008 3.1 respectively) after being identified through MegaBLAST searches and found to be present in P. syringae pv phaseolicola 1448a, but absent from P. syringae pv tomato DC3000 and Pseudomonas syringae pv syringae B728A; coding sequences were identified with NCBI's ORF Finder tool.

\section{Amino acid sequence analysis}

Each coding sequence annotated in the T3SS gene clusters of P. syringae pv phaseolicola 1448a, R. etli CIAT 652 and Rhizobium spp. NGR234 was analyzed by Psi-BLAST searches [10] against the NCBI non-redundant database reduced for bacteria using the following parameters: BLOSUM 65 substitution matrix; expected threshold 10; word size 3; gap costs: existence: 11 , extension 1; the filter for low complexity regions was set to on. The number of descriptions and alignments to be reported was set to 500 and conditional compositional adjustments were on. The program FoldIndex $\odot$ was used with default parameters for the prediction of structural disorder propensity from the amino acid sequences [11]. Secondary structure predictions were performed with PSIPRED [12]. Physical and chemical parameters of sequences under study were estimated by ProtParam [13]. Coiled coil predictions and assignment of the heptad repeat positions in proteins were produced in COILS [14] and MATCHER [15] respectively. Sequence threading techniques and fold-recognition algorithms were used to identify distant homologs. 3-D structural profiles for T3SS proteins were predicted from sequence data was performed using the PHYRE pipeline [16]. The program Memstat3 [17] was used for the prediction of membrane $\alpha$-helices in proteins.

\section{Nucleotide sequence analysis}

The gene synteny of the T3SS-2 clusters of $P$. syringae pv phaseolicola $1448 \mathrm{a}, P$. syringae pv oryzae str. $1 \_6, P$. syringae pv tabaci ATCC11528, Rhizobium spp. NGR234 and the gene synteny of the unique T3SS gene clusters of $B$. japonicum USDA $110, R$. etli CIAT 652, $R$. etli CNF 42, were compared to other known T3SS gene clusters of various bacteria using the BLASTN and BLASTP tools of the Genbank. Codon Usage Bias analysis was performed using DnaSP v5 [18].

\section{Phylogenetic analysis}

T3SS core protein sequences were retrieved using PsiBLAST searches with the $P$. syringae pv phaseolicola 1448a 
T3SS-2 gene cluster coding frames and were aligned with the multiple alignment method ClustalW, version 1.8 [19].

Phylogenetic relations were inferred using the neighbourjoining method [20] implemented in the MEGA4 software [21]. The bootstrap consensus tree inferred from 1000 replicates [22] is taken to represent the evolutionary history of the amino acid sequences analyzed [22]. Branches corresponding to partitions reproduced in less than $50 \%$ bootstrap replicates are collapsed. The percentage of replicate trees in which the associated taxa clustered together in the bootstrap test (1000 replicates) are shown next to the branches [22]. The tree is drawn to scale, with branch lengths in the same units as those of the evolutionary distances used to infer the phylogenetic tree. The evolutionary distances were computed using the Poisson correction method [23] and are in the units of the number of amino acid substitutions per site. All positions containing alignment gaps and missing data were eliminated only in pair wise sequence comparisons.

\section{Cultivation}

$P$. syringae strains were routinely grown at $28^{\circ} \mathrm{C}$ in LB medium. Bacteria of overnight culture were collected at an OD (optical density) of 0.8. The bacterial pellet was washed with $10 \mathrm{mM} \mathrm{MgCl}_{2}$ and the cells were resuspended (OD: 0.6-0.7) in Hrp-induction media [24] for overnight cultivation at $28^{\circ} \mathrm{C}$. The next day the bacterial cells were collected (OD: 0.7-0.8) for RNA extraction.

\section{RT-PCR}

For the RT-PCR reactions, total RNA was extracted from overnight bacterial cultures of $P$. syringae pv phaseolicola 1448a and P. syringae pv tomato DC3000, using both LB and Hrp-induction media [24]. Total RNA was treated with RNase-free DNase I for $45 \mathrm{~min}$ at $37^{\circ} \mathrm{C}$ [25]. From both culture conditions equal amounts of the extracted total RNA were subjected to RT-PCR with gene specific primers for the PSPPH_2530, PSPPH_2524 and $16 \mathrm{~S}$ rDNA genes, using the OneStep RT-PCR kit according to the manufacturer's instructions (QIAGEN). For negative control, PCR was performed on the total RNA without Reverse Transcriptase assay, using the $16 \mathrm{~S}$ rDNA primers, in order to accredit no DNA contamination in the total RNA isolation samples. The RT-PCR products were then analyzed by agarose gel electrophoresis. Primers sequences for $16 \mathrm{~S}$ RNA were $5^{\prime}$-CGGGTA CTTGTACCTGGTGGC-3' ${ }^{\prime}$ and $5^{\prime}$-CTTGCCAGTTTTG GATGCAGTTC-3', for PSPPH_2530 were $5^{\prime}$-AGGCCC TGACGACGCTGCTG-3' and 5'-CCAGGTGCCTGTG TTCGGCAGT-3' and for PSPPH_2524 5' $5^{\prime}$-TCCTGCTG TGCCTGTTATCCGGCG-3' and ${ }^{\prime}$-GACGGTCGGTAG CGACTTGAGTGAC-3'.

\section{Results and discussion}

Analysis of core components of $P$. syringae T3SS-2 and the Rhc-T3SS family

\section{Phylogenetic analysis of core proteins}

In the subsequent sections the unified nomenclature for T3SS proteins (Table 1) will be followed [26]. The phylogenetic analysis of various T3SS core proteins (including T3SS-2 proteins), e.g. SctU (RhcU/HrcU/YscU/FlhB and their homologues), SctV (RhcV/HrcV/LcrD/FlhA homolog proteins), SctQ (RhcQ/HrcQ/YscQ/FliN/ and their homologues) and the T3SS ATPases SctN (RhcN/HrcN/ YscN/FliI and homologues), confirmed the broad classification of the non-flagellar T3SS into seven families. However, the T3SS-2 proteins were grouped in the same clade with the Rhc T3SS proteins with high bootstrap values, suggesting that these lineages share a more recent common origin than with other T3SS families.

Interestingly, the Rhc T3SS family can be further subdivided into three subgroups: Subgroup I is represented by the well-known T3SSs of Rhizobium sp. NGR234, and B. japonicum USDA 110 while subgroup III is represented by the T3SS present in $R$. etli. Proteins from the T3SS-2 system of various $P$. syringae strains are grouped closer to the T3SS-2 of Rhizobium sp. NGR234 (Figure 1, 2, Additional files 1, Additional file 2 \& Additional file 3: Figures S1, S2 \& S3), forming the subgroup II of the Rhc T3SS family.

\section{All required core T3SS components are present in the T3SS- of $P$. syringae strains}

BLASTP and Psi-BLAST searches revealed the main T3SS components of the novel T3SS-2 gene cluster of $P$. syringae pv phaseolicola 1448 a which are also conserved in $P$. syringae $\mathrm{pv}$ oryzae str. $1 \_6, P$. syringae pv tabaci ATCC11528 (Additional file 4: Table S1) and P. syringae pv aesculi. Similar searches and comparisons were also carried out with the T3SSs of $R$. etli CNF 42, $R$. etli CIAT 652 and Rhizobium sp. strain NGR234. In the following, the prefix $\mathrm{Hrc}_{I I}$ will be used to specify the conserved T3SS-2 proteins of $P$. syringae pv phaseolicola 1448 a, $P$. syringae pv oryzae str. $1 \_6$ and $P$. syringae pv tabaci, while the prefix $\mathrm{Rhc}_{I I}$ will be used to distinguish the $R h c$ proteins of the T3SS-2 gene cluster found in plasmid pNGR234b of Rhizobium sp. NGR234 (see below). The T3SS protein nomenclature when used is indicated by the prefix Sct according to Table 1 .

All major T3SS core proteins were found in the T3SS gene clusters mentioned above, including the T3SS ATPase protein SctN (RhcN/HrcN/YscN/FliI homolog), its negative regulator SctL (NolV/HrpE/YscL/FliH homo$\log$ ), the two T3SS gate proteins SctU and SctV (RhcU/ $\mathrm{HrcU} / \mathrm{YscU} / \mathrm{FlhB}$ and RhcV/HrcV/LcrD/FlhA homologs respectively), the protein building the inner ring of the T3SS basal body SctJ (RhcJ/HrcJ/YscJ homolog), the protein building the cytoplasmic ring SctQ (RhcQ/HrcQ/ 
Table 1 T3SS proteins assigned under the unified nomenclature using the Sct (SeCreTion) prefix

\begin{tabular}{|c|c|c|c|c|c|c|c|c|c|c|c|c|c|c|c|c|c|c|}
\hline T3SS family & $\begin{array}{c}\text { Unified nomenclature vs } \\
\text { Species }\end{array}$ & SctV & SctW & SctN & SctO & SctP & SctQ & SctR & SctS & SctT & SctU & SctC & SctD & SctF & Sctl & SctJ & SctK & SctL \\
\hline \multirow[t]{2}{*}{ Ysc } & Yersinia sp. & LcrD & YopN & YscN & $\mathrm{YscO}$ & YsCP & YscQ & YscR & YscS & YscT & $\mathrm{YscU}$ & YscC & YscD & YscF & Yscl & YscJ & YscK & YsCL \\
\hline & $\begin{array}{l}\text { Pseudomonas } \\
\text { aeruginosa }\end{array}$ & PcrD & PopN & $\mathrm{PscN}$ & $\mathrm{PscO}$ & PsCP & PscQ & PscR & PscS & PscT & $\mathrm{PscU}$ & PscC & PscD & PscF & Pscl & PsCJ & PscK & PscL \\
\hline \multirow[t]{2}{*}{ Inv-Mxi-Spa } & Shigella flexneri & MxiA & Orf15MxiC & $\begin{array}{l}\text { SpaL } \\
\text { Spa47 }\end{array}$ & $\begin{array}{l}\text { SpaM } \\
\text { Spa13 }\end{array}$ & $\begin{array}{l}\text { SpaN } \\
\text { Spa32 }\end{array}$ & $\begin{array}{l}\text { SpaO } \\
\text { Spa33 }\end{array}$ & $\begin{array}{l}\text { SpaP } \\
\text { Spa24 }\end{array}$ & $\begin{array}{l}\text { SpaQ } \\
\text { Spa9 }\end{array}$ & $\begin{array}{l}\text { SpaR } \\
\text { Spa29 }\end{array}$ & $\begin{array}{l}\text { SpaU } \\
\text { Spa40 }\end{array}$ & MxiD & MxiG & MxiH & Mxil & MxiJ & MxiK & MxiN \\
\hline & Salmonella enterica & InvA & InvE & $\operatorname{lnvC}$ & Invl & InvJ & SpaO InvK & $\begin{array}{l}\text { SpaP } \\
\text { InvL }\end{array}$ & SpaQ & $\begin{array}{l}\text { SpaR } \\
\text { InvN }\end{array}$ & SpaS & $\operatorname{lnvG}$ & $\mathrm{PrgH}$ & Prgl & $\operatorname{Prg}\lrcorner$ & PrgK & OrgA & OrgB \\
\hline \multirow[t]{2}{*}{ Ssa-Esc } & Salmonella enterica & SsaV & & SsaN & $\mathrm{SsaO}$ & SsaP & SsaQ & SsaR & SsaS & SsaT & SsaU & SpiA SsaC & $\begin{array}{l}\text { SpiB } \\
\text { SsaD }\end{array}$ & SsaG & & SsaJ & & Ssak \\
\hline & EPEC & SepA & SepL & SepB & Orf15 & & SepQ & EscR & EscS & EscT & $\mathrm{EscU}$ & SepC & & ORFD2 & rOrf8 & SepD & & ORF5 \\
\hline Chlamydiales & $\begin{array}{l}\text { Chlamydia } \\
\text { trachomatis }\end{array}$ & CdsV & CopN & $\mathrm{CdsN}$ & $\begin{array}{l}\text { CT670 } \\
\text { CdsO }\end{array}$ & $\begin{array}{l}\text { CT671 } \\
\text { CdsP }\end{array}$ & $\mathrm{Cds} \mathrm{Q}$ & CdsR & $\mathrm{CdsS}$ & CdsT & $\mathrm{Cds} U$ & $\mathrm{Cds} C$ & $C d s D$ & CsdF & & CdsJ & & CdsL \\
\hline \multirow[t]{2}{*}{ Hrp-Hrc1 } & $\begin{array}{l}\text { Pseudomonas } \\
\text { syringae }\end{array}$ & $\mathrm{HrcV}$ & HrpJ & $\mathrm{HrCN}$ & HrpO & HrpP & $\begin{array}{l}\operatorname{HrcQ}_{A} \& \\
\mathrm{HrcQ}_{B}\end{array}$ & $\mathrm{HrcR}$ & $\mathrm{HrCS}$ & HrcT & $\mathrm{HrCU}$ & $\mathrm{HrcC}$ & HrpQ & HrpA & $\mathrm{HrpB}$ & $\mathrm{HrcJ}$ & HrpD & HrpE \\
\hline & Erwinia amylovora & $\mathrm{HrCV}$ & HrpJ & $\mathrm{HrCN}$ & $\mathrm{HrpO}$ & HrpP & HrcQ & $\mathrm{HrcR}$ & $\mathrm{HrCS}$ & HrcT & $\mathrm{HrcU}$ & $\mathrm{HrcC}$ & HrpQ & HrpA & $\mathrm{HrpB}$ & $\mathrm{HrCl}$ & & HrpE \\
\hline \multirow[t]{3}{*}{ Hrp-Hrc2 } & $\begin{array}{l}\text { Burkcholderia } \\
\text { pseudomallei }\end{array}$ & SctV & & SctN & HrpD & $\mathrm{HpaC}$ & SctQ & SctR & SctS & SctT & SctU & SctC & SctD & & & Sct & & SctL \\
\hline & $\begin{array}{l}\text { Ralstonia } \\
\text { solanacearum }\end{array}$ & $\mathrm{HrcV}$ & & $\mathrm{HrCN}$ & HrpD & $\mathrm{HpaP}$ & $\mathrm{HrcQ}$ & $\mathrm{HrcR}$ & $\mathrm{HrCS}$ & HrcT & $\mathrm{HrcU}$ & $\mathrm{HrcC}$ & HrpW & HrpY & HrpJ & $\mathrm{HrCJ}$ & & $\mathrm{HrpF}$ \\
\hline & $\begin{array}{l}\text { Xanthomonas } \\
\text { campestris }\end{array}$ & $\mathrm{HrcV}$ & НpaA & $\mathrm{HrCN}$ & HrpB7 & $\begin{array}{l}\mathrm{HpaP} \\
\mathrm{HpaC}\end{array}$ & $\mathrm{HrcQ}$ & $\mathrm{HrcR}$ & $\mathrm{HrCS}$ & HrcT & $\mathrm{HrcU}$ & $\mathrm{HrcC}$ & HrpD5 & HrpE & HrpB2 & $\mathrm{HrCJ}$ & & HrcLHrpB5 \\
\hline \multirow[t]{4}{*}{ Rhc } & $\begin{array}{l}\text { Subgroup I Rhizobium } \\
\text { pNGR234a }\end{array}$ & Y4yR & - & $\mathrm{RhcN}$ & Y4y」 & - & RhcQ & RhcR & RhcS & RhcT & RhcU & $\begin{array}{l}\text { NolW RhcC } \& \\
\operatorname{RhcC}_{2}\end{array}$ & Y4yQ & & NolU & NolT & & NolV \\
\hline & $\begin{array}{l}\text { Subgroup II P. } \\
\text { syringae }\end{array}$ & $\mathrm{Hrc}_{/ 1} \mathrm{~V}$ & & $\mathrm{HrC}_{/ /} \mathrm{N}$ & $\mathrm{Hrc}_{/ /} \mathrm{O}$ & & $\mathrm{Hrc}_{/} \mathrm{Q}$ & $\mathrm{Hrc} / / R^{R}$ & $\mathrm{Hrc}_{\|} \mathrm{S}$ & $\mathrm{Hrc}_{/ l} \mathrm{~T}$ & $\mathrm{HrC}_{/ /} \mathrm{U}$ & $\mathrm{HrC}_{\|} \mathrm{C}_{1} \mathrm{Hrc}_{\|} \mathrm{C}_{2}$ & $\operatorname{Hrp}_{/} \mathrm{Q}$ & & & $\mathrm{Hrc}_{\mid / J}$ & & $\mathrm{Hrp}_{/ / \mathrm{E}}$ \\
\hline & $\begin{array}{l}\text { Subgroup II } \\
\text { Rhizobium pNGR234b }\end{array}$ & $\mathrm{Rhc}_{/ /} \mathrm{V}$ & - & & $\mathrm{Rhc}_{/ /} \mathrm{O}$ & - & $\mathrm{Rhc}_{\|} \mathrm{Q}$ & $\mathrm{Rhc}_{\mu} \mathrm{R}$ & $\mathrm{Rhc}_{\| / S}$ & $\mathrm{Rhc}_{\| /} \mathrm{T}$ & $\mathrm{Rhc}_{\|} \mathrm{U}$ & $\begin{array}{l}\operatorname{Rhc}_{\|} C_{1} \& \\
\operatorname{Rhc}_{\|} C_{2}\end{array}$ & $\mathrm{Rhp}_{/ / \mathrm{Q}}$ & & & & & $\mathrm{Rh}_{/ / /} \mathrm{L}$ \\
\hline & $\begin{array}{l}\text { Subgroup III } \\
\text { Rhizobium etli }\end{array}$ & RhcV & - & RhcN & RhcO & - & RhcQ & RhcR & RhcS & RhcT & RhcU & $\mathrm{RhcC}_{1}$ & & & NolU & RhcJ & & RhcL \\
\hline Flagellar & & FlhA & & Flil & FliJ & & $\begin{array}{l}\text { FliY FliM \& } \\
\text { FliN }\end{array}$ & Flip & FliQ & FliR & FlhB & & FliG & & & FliF & & $\mathrm{FliH}$ \\
\hline
\end{tabular}



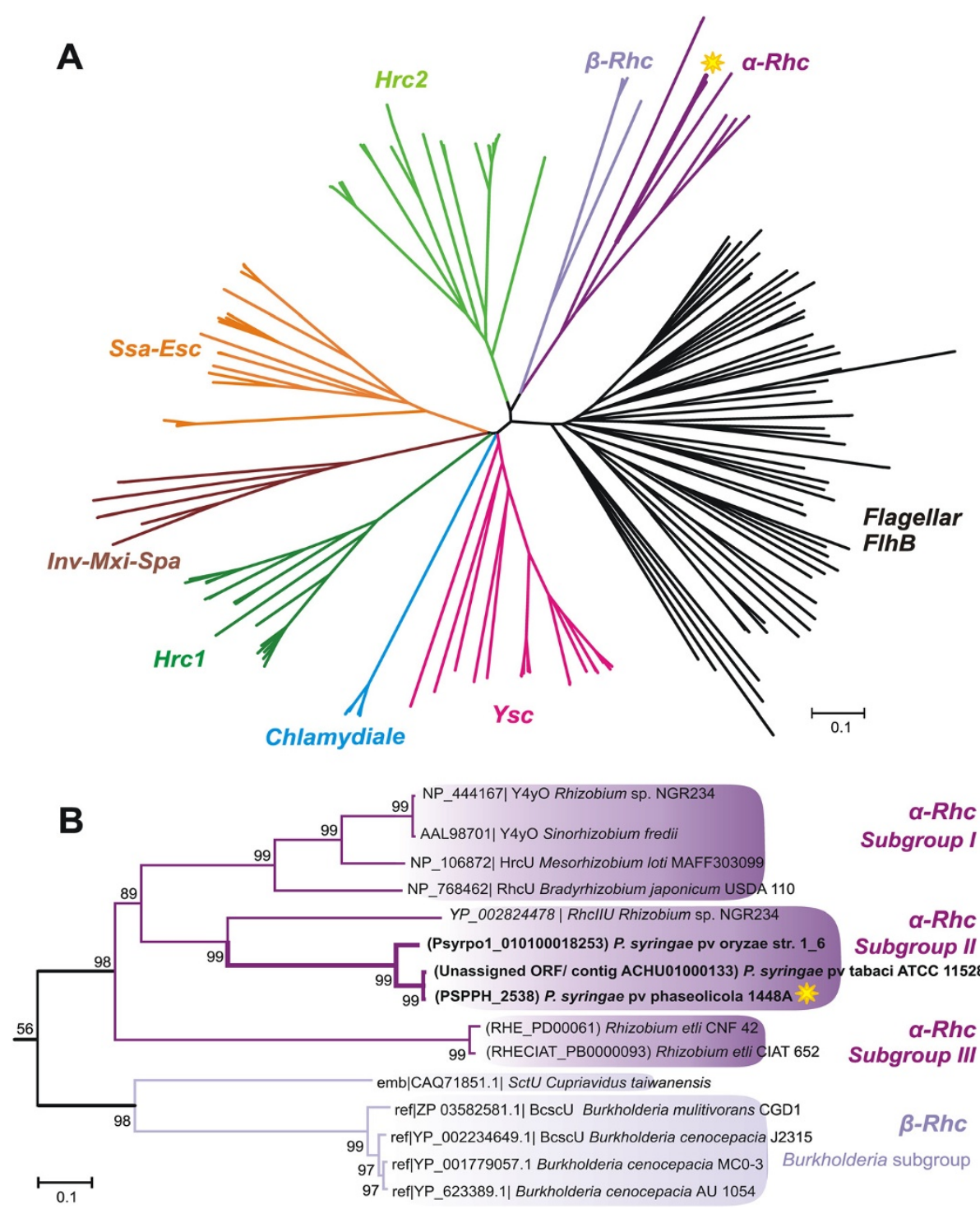

Figure 1 Evolutionary relationships of SctU proteins. The yellow star indicates the position of the $P$. syringae pv phaseolicola 1448 a $\mathrm{Hrc} / \mathrm{U}$. A. The phylogram of $192 \mathrm{SctU}$ sequences with the eight main families named according to Troisfontaines \& Cornelis (2005) [8], while the flagellum proteins are depicted in black. The T3SS family encompasing the $\beta$-rhizobium Cupriavidus taiwanensis and of Burkholderia cenocepacia group is indicated here with a light purple color (marked as $\beta$-Rhc). Branches corresponding to partitions reproduced in less than $50 \%$ bootstrap replicates are collapsed. There were a total of 686 positions in the final dataset. Phylogenetic analyses were conducted in MEGA4 [21]. B. The Rhc T3SS clade as derived from the phylogram in A, groups the P. syringae Hrc $/ U$ sequences close to the Rhc $U$ protein of the Rhizobium sp. NGR234 T3SS-2. The values at the nodes are the bootstrap percentages out of 1000 replicates. The locus numbers or the protein accession number of each sequence is indicated.

YscQ/FliY homolog) and the three core membrane proteins SctR, SctS, SctT (RhcRST/HrcRST/YscRST/FliPQR homologs) (Additional file 4: Table S1).

It is noteworthy that the promoter regions of the T3SS-2 ORFs/operons of $P$. syringae pv phaseolicola 1448a, do not appear to harbor "hrp box" elements like those which have been described for the T3SS-1 genes of various $P$. syringae strains [27]. This, coupled with the low expression level seen in minimal media (Figure 3), leave open the question whether T3SS-2 in this or other $P$. syringae strains is expressed under in planta conditions and whether it is plays a role in their phytopathogenic potential or in any other aspect of their life cycle.

\section{The split secretin gene}

A distinguishing feature of gene organization in $R h c$ T3SS clusters is a split gene coding for the outer membrane secretin protein $\mathrm{SctC}$, i.e. a $\mathrm{HrcC} / \mathrm{YscC}$ homologue [28]. This is also true for the subgroup II $R h c$ T3SS gene 


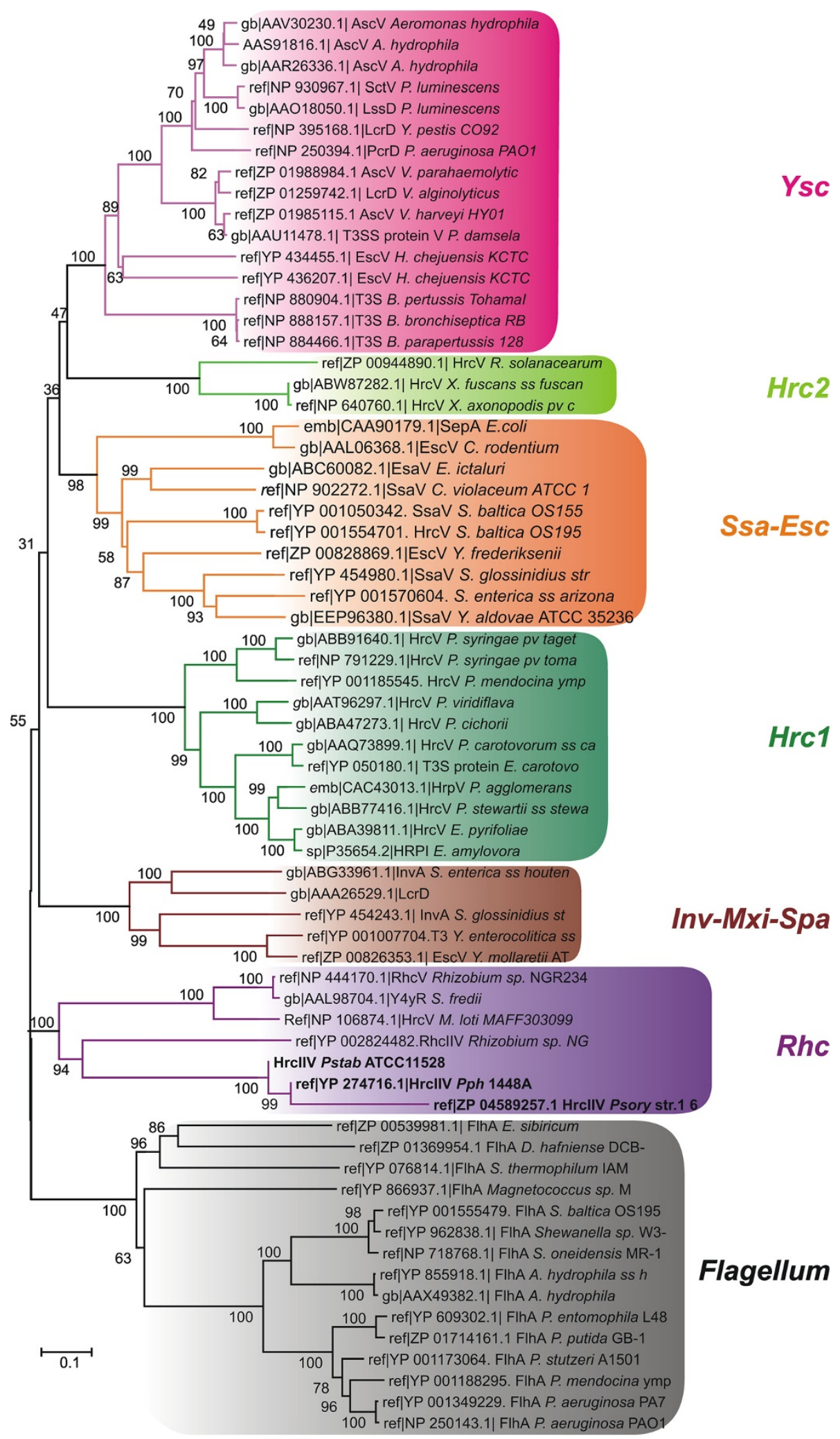

Figure 2 Evolutionary relationships of SctV proteins. Classification of the SctV T3SS proteins into the main T3SS/flagellar families. The colouring scheme of Figure 1 is used. 


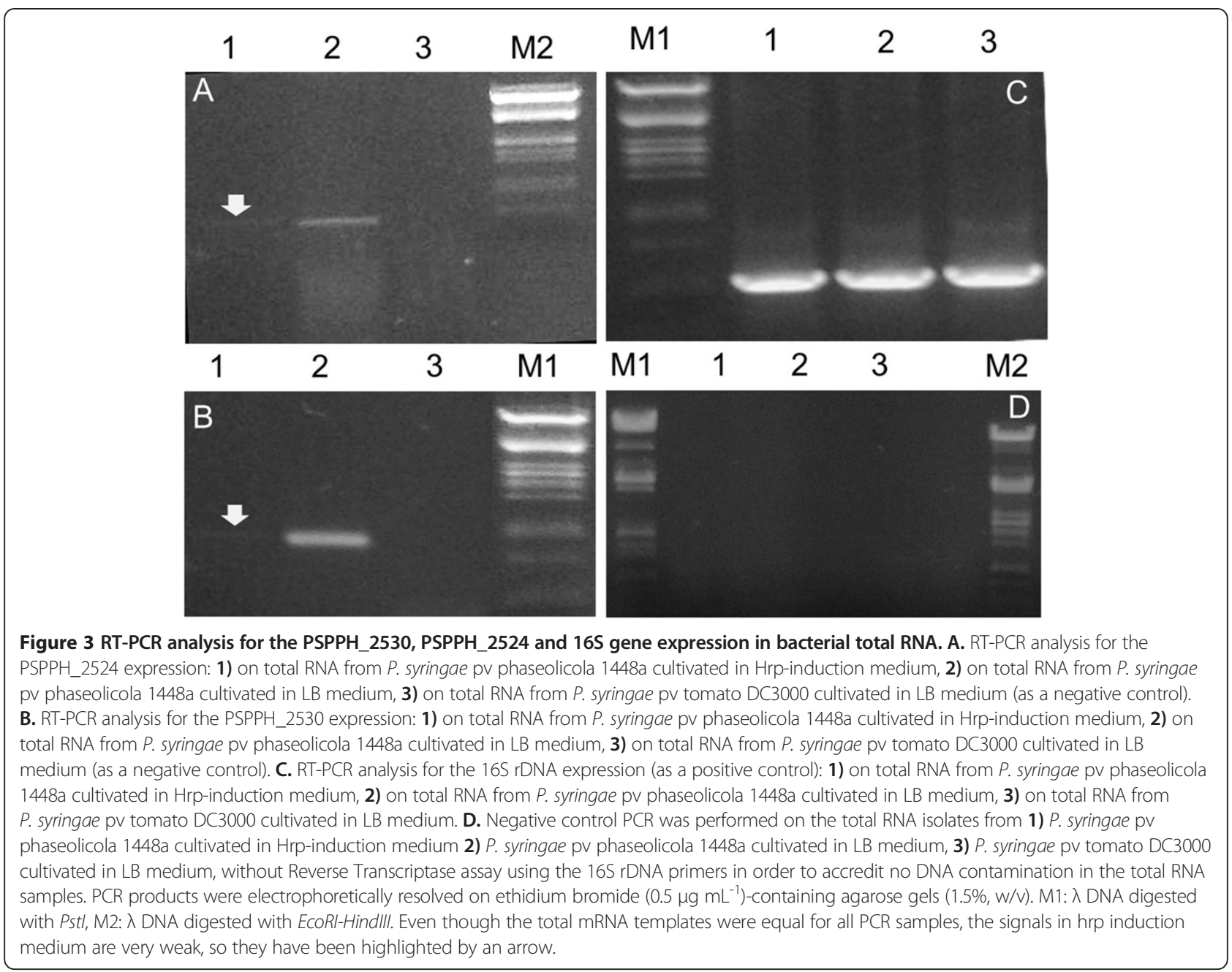

clusters. In the T3SS-2 clusters of the three $P$. syringae pathovars the secretin gene is split in two ORFs (Figure 4, Additional file 4: Table S1). In P. syringae pv phaseolicola 1448a, loci PSPPH_2524 $\left(h r c_{I I} C 1\right)$ and PSPPH_2521 $\left(h r_{I I} C 2\right)$ code for the $\mathrm{N}$-terminal and the C-terminal part of secretin, respectively, of a $\mathrm{HrcC} / \mathrm{YscC}$ homolog. Comparisons of $\mathrm{Hrc}_{I I} \mathrm{C} 1$ and $\operatorname{Hrc}_{I I} \mathrm{C} 2$ with the RhcC1 and Rhc2 proteins of Rhizobium sp. NGR234 are given in Additional file 5: Figure S4, respectively. A similar situation occurs in $P$. syringae pv oryzae str. 1_6 while in $P$. syringae pv tabaci ATCC11528 $\operatorname{Hrc}_{I I} C 2$ gene is further split into two parts. However in P. syringae pv phaseolicola 1448a and P. syringae pv tabaci ATCC11528 the two $h r c_{I I} C 1, h r c_{I I} C 2$ genes are only separated by an opposite facing ORF coding for a TPR-protein, while in the subgroup I Rhc T3SS these two genes are separated even further (Figure 4). Although the functional significance of the split secretin gene is not known, there are reports of constitutive expression of the $r h c C 1$ gene in contrast to the rest of the T3SS operons in rhizobia $[29,30]$. In subgroup III only the $r h c C 1$ could be identified (RHECIAT_PB0000097 in the R. etli CIAT 652 and RHE_PD00065 in $R$. etli CNF 42) in Psi-BLAST searches using the $\mathrm{Hrc}_{I I} \mathrm{C} 1$ protein sequence as query (25\% identity to RhcC1 of Rhizobium sp. NGR234) (Figure 4).

\section{The Hrc ${ }_{1 /} Q$ protein}

The PSPPH_2534 locus (designated $h r c_{I I} Q$ ) in the T3SS-2 cluster of $P$. syringae pv phaseolicola 1448A codes for a polypeptide chain of 301 residues, which has sequence similarities with members of the HrcQ/ YscQ/FliY family. Members of this family usually consist of two autonomous regions [26] which either are organized as two domains of a single protein or can be split up into two polypeptide chains. The $\operatorname{Hrc}_{I I} \mathrm{Q}$ is comparable in length with the long proteins of the family. The same is true in the Rhc-T3SS case, where an HrcQ ortholog is found. In agreement with the other $\mathrm{HrcQ} / \mathrm{YscQ} / \mathrm{FliY}$ members the sequence conservation is especially high at the C-terminus [31,32]. In the originally described T3SS-1 (Hrc-Hrp1) of P. syringae strains this gene is split into two 
adjacent ORFs coding for separate polypeptides $\left(\mathrm{HrcQ}_{\mathrm{A}}\right.$ and $\left.\mathrm{Hrc}_{\mathrm{B}}\right)$. No splitting occurs however in the T3SS-2 clusters of the P. syringae strains.
The HrpO-like protein

A conserved feature in gene organization of T3SS gene clusters and the flagellum is the presence of a small ORF

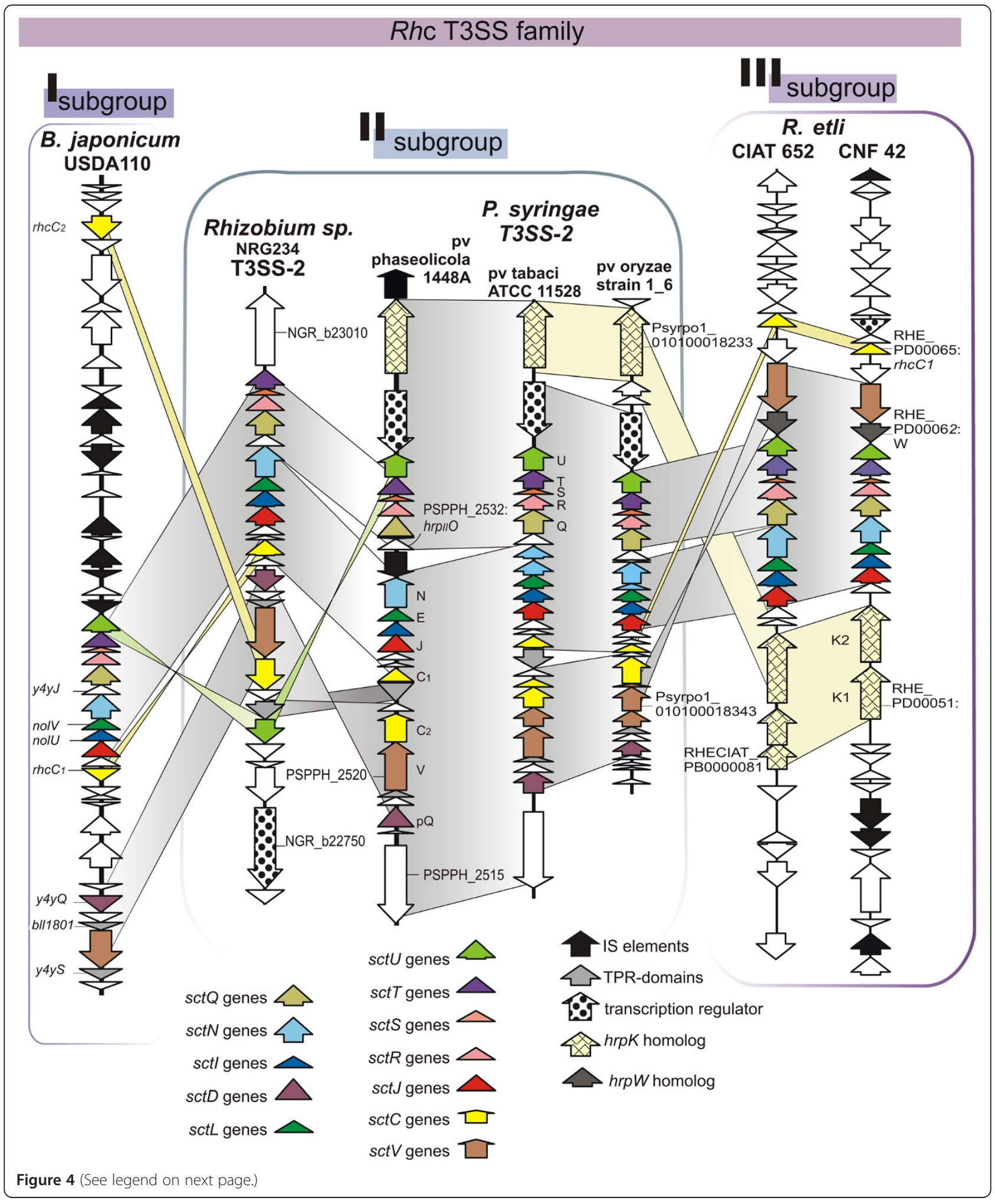


(See figure on previous page.)

Figure 4 Genetic organization of the Rhc T3SS gene clusters, indicating the diversification of three main subgroups. ORFs are represented by arrows. White arrows indicate either low sequence similarities between syntenic ORFs like the PSPPH_2532: hrpO/l case or ORFs not directly related to the T3SS gene clusters that were excluded from the study. Homologous ORFs are indicated by similar coloring or shading pattern. Only a few loci numbers are marked for reference. Gene symbols ( $\mathrm{N}, \mathrm{E}$, J etc.) for the T3SS-2 genes are following the Hrc1 nomenclature. 1) Subgroup I cluster (Rhc-I), is represented by Bradyrizhobium japonicum USDA110 and includes also the T3SS present on the pNGR234a plasmid of strain NGR234 (not shown); 2) Subgroup II (Hrc/VRhc/l), represented by the T3SS-II gene clusters of Rhizobium sp. NGR234 pNGR234b plasmid [38] , P. syringae pv phaseolicola 1448A [44], P. syringae pv tabaci ATCC 11528 and P. syringae pv oryzae str. 1_6 (this study, see Materials and Methods); and 3) subgroup III, represented by the sole T3SS of the Rhizobium etli CIAT652 (plasmid b) and the R. etli CNF42 plasmid d [37]. Gene

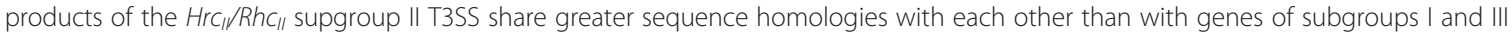
(Additional file 4: Table S1).

downstream of the gene coding for the ATPase (hrcN/ yscN/fliI homologue). These ORFs code for proteins of the $\mathrm{HrpO} / \mathrm{YscO} / \mathrm{FliJ}$ family, a diverse group characterized by low sequence similarity, and heptad repeat motifs suggesting a high tendency for coiled-coil formation and a propensity for structural disorder [33]. Such a gene is also present in the Rhizobium NGR234 T3SS-2 but is absent from the subgroup III $R h c$-T3SS where the $r h c Q$ gene is immediately downstream of the $r h c N$ gene (Figure 4). In the P. syringae pathovars included in Figure 4 there is a small ORF (PSPPH_2532 in strain P. syringae pv phaseolicola 1448A, Figure 4) coding for a polypeptide wrongly annotated as Myosin heavy chain B (MHC B) in the NCBI protein database. Sequence analysis of this protein and its homologs in the other two P. syringae strains using BLASTP searches did not reveal any significant similarities to other proteins. However, these small proteins are predicted as unfolded in their entire length, while heptad repeat patterns are recognizable in the largest part of their sequence, thus strongly resembling the properties of members of the HrpO/YscO/FliJ family [33], (Additional file 6: Figure S5). A potentially important feature in the P. syringae pv phaseolicola 1448a T3SS-2 cluster is a predicted transposase gene between the ORF coding for the above described $\mathrm{HrpO} / \mathrm{YscO} / \mathrm{FliJ}$ family member and the ORF for the $\operatorname{Hrc}_{\mathrm{II}} \mathrm{N}$ ATPase (Figure 4); this gene is absent from the $P$. syringae pv tabaci and P. syringae pv oryzae str.1_6 T3SS-2 clusters. The insertion of the transposase gene does not disrupt genes $h r c_{I I} N$ or $h r p_{I I} O$ as concluded by amino acid sequence comparison with other members of the SctN and SctO protein families respectively (including ORFs from other T3SS-2 P. syringae strains). These genes are capable of producing the respective full-length proteins and no premature termination, due to transposase insertion, is observed.

\section{The HrpQ-like protein}

Another common feature of P. syringae T3SS-2 and the Rhizobium T3SSs excluding subgroup III, is a gene usually positioned upstream of the $s c t V$ gene $(r h c V / h r c V /$ lcrD/flhA homolog) and in close proximity to it. Psi-
BLAST searches for the PSPPH_2517 encoded protein revealed moderate similarities to the $\mathrm{HrpQ} / \mathrm{YscD}$ family of T3SS proteins; these were confirmed by sequence threading techniques. For example, a segment of of PSPPH_2517 corresponding to $45 \%$ of its amino acid sequence scores an E-value of 2e-05 and a 26\% identity with YscD protein from Yersinia enterocolitica (ref) YP_006007912.1); the same segment scores an E-value of $1 \mathrm{e}-13$ with $25 \%$ identity to the $90 \%$ of its sequence with the equivalent protein from B. japonicum USDA110 (ref| NP_768443.1). The chosen folding templates belong to various forkhead - associated (FHA) protein domains from different origins. FHA cytoplasmic domains characterize the YscD/EscD protein family and may suggest phosphopeptide recognition interactions [34]. A protein with the above characteristics is present in the B. japonicum USDA110 T3SS cluster (encoded by the $y 4 y Q$ gene) while an ortholog could not be identified in the R. etli T3SS.

\section{Gene clusters organization in the Rhc-T3SS family and the P. syringae T3SS-2}

Subgroup I of the Rhc-T3SS family comprises the first described and well characterized T3SS-1 of Rhizobium NGR234 present in the plasmid pNGR234a [35], along with that of B. japonicum USDA110 and others [36]. The T3SS core genes in this case are organized in three segments. The biggest segment harbors the genes rhcU, rhcT, rhcS, rhcR, rhcQ, y4yJ, rhcN, nolV, nolU, rhcJ, nolB, in the same DNA strand with the $r h c C 1$ gene flanking the nolB gene in the opposite strand (Figure 4, Subgroup I). The second one harbors the $r h c V$ gene usually between the $y 4 y S$ and $y 4 y Q$ genes, all in the same orientation. In the case of the B. japonicum USDA110 however there are two additional open reading frames (ORFs) between the $r h c V$ and the $y 4 y Q$ gene in the same orientation (Figure 4, Subgroup I). The third segment harbors the $r h c C 2$ gene usually between the $y 4 x I$ and the $y 4 x K$ genes.

Subgroup III of the $R h c$-T3SS family includes the T3SS of $R$. etli strains CIAT652 (plasmid b) and CNF42 (plasmid d) [37]. The gene organization is very different from 
that of subgroup I in that there is no $r h c C 2$ gene, while the $r h c V$ gene is in close proximity to the biggest segment. In the biggest segment the genes $y 4 y \mathrm{~J}(\mathrm{hrpO} / \mathrm{yscO} / \mathrm{fliJ}$ homolog) and $n o l B$ are missing. Additional genes present in the subgroup III are coding for a HrpK-like protein (hypothetical translocator of the Hrc-Hrp1 T3SS) and a HrpW-like protein.

Gene clusters of subgroup II of the Rhc T3SS family, represented by the the T3SS-2 of Rhizobium NGR234 (pNGR234b plasmid) [38] and the recently identified T3SS-2 gene clusters of the P. syringae, possesses various characteristics that classify them as intermediates between the T3SS subgroups I and III. On one hand, subgroup II clusters share the $s c t O, s c t D$ and $s c t C 2$ genes with subgroup I clusters and but not with subgroup III; on the other hand, some subgroup II clusters posses putative translocator genes present in subgroup III, but absent from subgroup I.

The T3SS-2 clusters of the P. syringae strains are essentially syntenic, with the exceptions of an IS element (insertion sequence element) being present between the $\mathrm{Hrc}_{I I} \mathrm{~N}$ and $\operatorname{Hrp}_{I I} \mathrm{O}$ coding frames in the P. syringae pv phaseolicola 1448a cluster and the absence of a TPR (tetratricopeptide repeats) protein coding frame in the P. syringae pv oryzae str.1_6 cluster. The Rhizobium sp. NGR234 pNGR234b-plasmid borne cluster has two extended regions of synteny with those of the P. syringae strains. One is the region from $\operatorname{hrc}_{I I} C_{1}$ to $\operatorname{hrc}_{I I} T$, [not including the IS element in the P. syringae pv phaseolicola 1448a cluster (see above)]. The other is the region from $h r p_{I I} Q$ to $P S P P H \_2522$ which, however, is inverted in the Rhizobium sp. NGR234 pNGR234b T3SS cluster relative to those in the pseudomonads. The coding frame for the $\mathrm{RhcU} / \mathrm{HrcU} / \mathrm{YscU} / \mathrm{FhlB}$ homolog in the NGR234 cluster is transposed in relation to the Pseudomonas cluster (position which is maintained in the R.etli and B. japonicum clusters). In subgroup II of Rhc-T3SS gene clusters an $h r c_{I I} C 2$ gene can be identified in synteny to the subgroup I cluster. A common property of subgroups II and III of $R h c$-T3SS gene clusters is the presence of $h r p K$-like genes.

Common to all $R h c$-T3SS subgroups is the absence of a $h r p P / y s c P$-like gene which usually resides between the $h r p O / y s c O$-like gene and the $h r c Q / y s c Q$ homolog gene. A $h r p O / y s c O-l i k e$ gene is absent from the subgroup III cluster. Subgroup I and III clusters maintain synteny with the $P$. syringae T3SS-2 clusters for most of the core T3SS ORFs. Finally, a gene coding for a HrpW homolog is found only in the $R$. etli clusters.

\section{Non-conserved T3SS proteins}

The translocator of the P. syringae T3SS-2

A common feature of the $R$. etli Rhc T3SS (subgroup III) and the T3SS-2 of P. syringae pathovars (but not of the
Rhizobium sp. NGR234 T3SS-2) is the presence of an ORF coding for a hypothetical translocator protein: The PSPPH_2540 locus of the P. syringae pv phaseolicola 1448a T3SS-2 codes for a large protein of 1106 residues. The C-terminal part of this protein (residues 421 1106) is homologous to the HrpK proteins of the HrcHrp1 T3SS family based on Psi-BLAST searches (25\% identity with HrpK of Erwinia amylovora). HrpK shares low similarity with the putative translocator, HrpF, from Xantomonas campestris pv vesicatoria. Furthermore, the C-terminal part of the protein coded by PSPPH_2540 also possesses two predicted transmembrane $\alpha$-helices comprising residues 879-898 and 1029-1047 (MEMSAT3 analysis). The subgroup I Rhc T3SS lacks a $h r p K$ ortholog. The HrpK protein was initially identified as a component of the Hrc-Hrp1 family of T3S systems [39]. Interestingly, the $R$. etli T3SS gene cluster possesses two copies of $h r p K$-like genes, plus an additional $h r p W$-like gene, coding for an Hrp-secreted protein homologous to class III pectate lyases which is absent from the P. syringae pv phaseolicola 1448a T3SS-2 gene cluster but present in the extremity of the Hrc-Hrp1 gene cluster of P. syringae pv phaseolicola 1448a. These differences possibly suggest variations in the mode of interaction of these bacteria with their hosts.

\section{The two unknown ORFs upstream of the rhcV gene in subgroup II Rhc-T3SS gene clusters}

The choice of the B. japonicum USDA 110 T3SS as archetypal for subgroup I in the $R h c$ family (Figure 4) and for synteny comparisons with the subgroup II gene clusters, was based on the DNA segment encompassing rhcV $(y 4 y Q-y 4 y S)$. The presence of two small open reading frames upstream of the $r h c V$ gene and downstream of the $y 4 y Q$ gene of the known Rhizobium T3SS resembled the case of the $P$. syringae pv phaseolicola 1448a T3SS-2 where loci PSPPH_2518 and PSPPH_2519 are found between the ORF coding for the SctV protein (RhcV/HrcV/LcrD/FlhA homolog) and the ORF coding for the SctD protein (HrpQ/YscD homolog).

The PSPPH_2519 locus, upstream of the $h r c_{I I} V$ gene of $P$. syringae pv phaseolicola 1448a genome, encodes for a 112 long polypeptide with sequence similarities to the VscY protein of Vibrio parahaemolyticus, according to Psi-BLAST searches $(\mathrm{E}$-value $=0.005)$. The $v s c Y$ gene is located upstream of the $v c r D$ gene and this synteny is also conserved in the Ysc T3SS gene cluster family. Proteins YscY, VscY and PSPPH_2519 all possess TPR repeats (Tetratricopeptide Repeats) as predicted by PsiBLAST searches and fold recognition methods. YscY has been found to directly bind the YscX protein, a secreted component of the Ysc T3SS [40]. The bll1801 locus of B. japonicum USDA110 encodes for a 142 long polypeptide with TPR repeats and sequence similarities to the 
AscY (Aeromonas salmonicida) and YscY proteins according to Psi-BLAST searches. The position of bll1801 is likewise upstream of the $r h c V$ gene in B. japonicum USDA110 T3SS gene cluster. A protein with the above characteristics could not be identified for the R. etli T3SS (subgroup III), however it is present in the T3SS-2 of Rhizobium NGR234.

\section{Transcription regulators in P. syringae T3SS-2}

The Hrc-Hrp2 and the Rhc T3S (subgroup I) systems possess transcription regulators that belong to the $\mathrm{AraC} /$ XylS in contrast to the Hrc-Hrp1 T3SS that depends on the alternative sigma factor HrpL. The known transcription factors are related to the T3SS regulation of AraC and LuxR/UhaP families of transcription regulators and characterized by two $\alpha$-helix-turn- $\alpha$-helix (HTH) motifs in a tetrahelical bundle.

However, the PSPPH_2539 locus of P. syringae T3SS-2 codes for a hypothetical transcription regulator with different characteristics. The N-terminal part of the hypothetical protein (Figure 5, blue-purple area) is predicted to adopt a structure similar to the DNA-binding domains of the PhoB transcription factor. The characteristic $\mathrm{HTH}$ motif is a common feature of transcription factors. Although the PSPPH_2539 ORF is annotated in the NCBI as a LuxR-type of transcription regulator, the choice of the DNA-binding domain of $\mathrm{PhoB}$ as a structural template indicates that PSPPH_2539 probably has an $\alpha-/ \beta$ - doubly wound fold (distinguished by the presence of a $\mathrm{C}$-terminal $\beta$-strand hairpin unit that packs against the shallow cleft of the partially open tri-helical HTH core) motif. Transcription factors are usually multidomain proteins, thus the assignment of PSPPH_2539 as a LuxR-type transcription regulator in the NCBI is probably due to full-length inadequate Psi-BLAST searches biased by the presence of Tetratricopeptide Repeats (TPR) in the large carboxyterminal domain.

The middle part of the protein (Figure 5, yellow area) was found homologous to the $\mathrm{AAA}^{+}$ATPases (COG3903) based on fold-recognition algorithms and Psi-BLAST searches. These ATPases are associated with diverse cellular activities and are able to induce conformational changes in their targets [41]. In the context of the transcription process, $\mathrm{AAA}^{+}$ATPase domains are involved in the remodeling of $\sigma^{54}$ RNA polymerases. Especially the residues 195 to 300 probably possess the receiver or ligand binding domain of the hypothetical transcription factor (green area, Figure 5).

\section{TPR-repeats proteins present in P. syringae T3SS-2}

Apart from the PSPPH_2539 C-terminal domain, there are two more ORFs, PSPPH_2519 and PSPPH_2523, from the $P$. syringae pv phaseolicola 1448 a T3SS-2 that are predicted to code for proteins that possess TPR domains. TPR domains are typically found in class II chaperones of T3S systems - chaperones of the translocators - as well as in transcriptional regulators of the T3S systems, e.g. the HrpB protein of Ralstonia solanacearum, HilA of Salmonella enterica [42] and SicA, of Salmonella typhimurium involved in the activations of

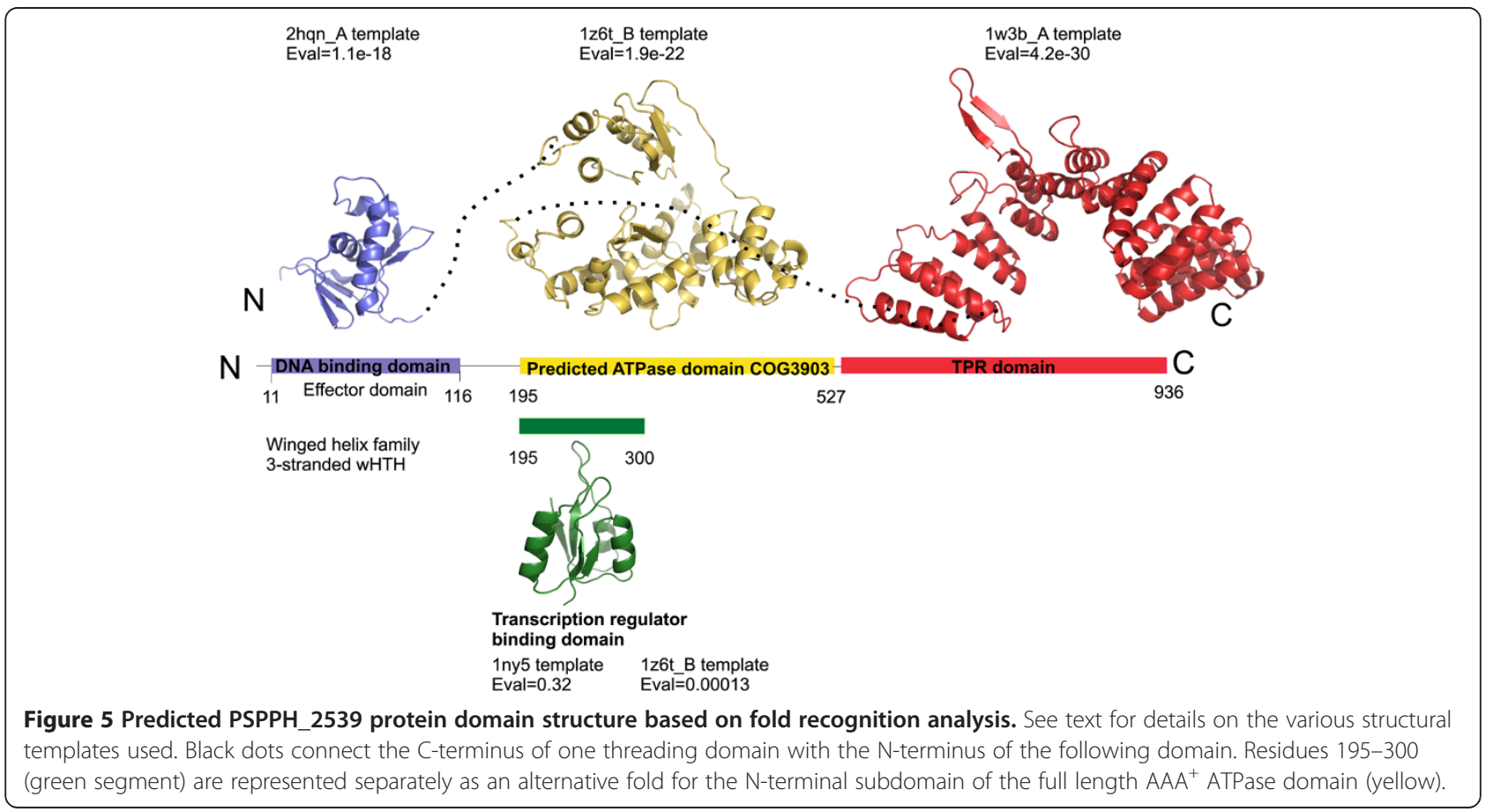


T3SS virulence genes [43]. Proteins with TPR repeats also exist in the Hrc-Hrp2 T3S system of X. campestris (HrpB2 protein) and in the T3S system of Rhizobia (e.g. the 182 residue long Y4yS protein). On the other hand, the Hrc-Hrp1 system of P. syringae does not possess proteins with TPR repeats.

DNA characteristics of the $P$. syringae T3SS-2 gene cluster The T3SS-2 cluster of $P$. syringae pv phaseolicola 1448a is separated by $1.42 \mathrm{Mb}$ from the well-characterized Hrc-Hrp1 T3SS cluster in the main chromosome. Both clusters are located on DNA segments with GC content similar to their neighbouring areas. No sequences associated with HrpL-responsive promoters (characteristic for the regulation of the Hrc-Hrpl operons in P. syringae pathovars) were found in the T3SS-2 gene cluster [44] indicating a different way of regulation from the Hrc-Hrp1 system. The ORF PSPPH_2539 that resides between the core genes and the hrpK homolog PSPPH_2540, codes for a hypothetical transcription regulator (Figure 4, 5). No $t_{R N A}$ genes, however, have been found in the vicinity of this cluster, while two insertion sequence (IS) elements occur in the border and in the middle region of the T3SS2 gene cluster (Figure 4).

The GC content of the T3SS-2 cluster in the P. syringae strains is close to the chromosome average (58-61\%), which might suggest that it has been resident in the $P$. syringae's genome for a long time [45]. The codon usage indexes (Additional file 7: Table S2) of the T3SS-2 cluster show the same degree of codon usage bias as the hrc-hrp1 T3SS cluster of P. syringae pv phaseolicola 1448a. Furthermore, the GC content in the third coding position (GC3) of various genes across the T3SS- 2 is close to the respective mean of the genome GC3, as in the case of Hrc-Hrp1 (Additional file 7: Table S2). These equal GC levels could indicate an ancient acquisition of the T3SS-2 gene cluster by $P$. syringae that was lost in some of its strains. However the scenario of a more recent acquisition from a hypothetical donor with equal GC levels can not be excluded.

\section{Evidence for expression of the $P$. syringae T3SS-2}

There are no reports so far for the expression or function of T3SS-2 in members of P. syringae. To obtain preliminary expression evidence of functional putative RNA transcripts, the $h r c_{I I} N(s c t N)$ and $h r c_{I I} C 1$ (sctC) from $P$. syringae pv phaseolicola 1448 a were detected by RTPCR in total RNA extracts from cultures grown in rich (LB) and minimal (M9) media, after exhaustive treatment with RNase-free DNase I (Supplier Roche Applied Science). Putative transcripts were detected under both growth conditions that were tested, using equal amounts of the extracted total RNA as an RT-PCR template. Interestingly, the detected transcript levels were remarkably higher in LB medium (Figure 3), compared to minimal (M9) medium, probably indicating that the genes are expressed in both cultivation conditions.

\section{Conclusions}

Rhizobia are $\alpha$-proteobacteria that are able to induce the formation of nodules on leguminous plant roots, where nitrogen fixation takes place with T3SS being one important determinant of this symbiosis $[36,46,47]$. Sequences of the symbiotic plasmids of Rhizobium strains NGR234 and $R$. etli CFN42 together with the chromosomal symbiotic regions of B. japonicum USDA110 and Mesorhizobium loti R7A have been recently reported [36-38]. An unusual feature of the Rhizobium strains NGR234 [38], is the presence of an additional T3SS gene cluster.

Members of the $P$. syringae species are gram negative plant-associated $\gamma$-proteobacteria that can exist both as harmless epiphytes and as pathogens of major agricultural crops [48-52]. Pathogenic varieties of this species utilize a Hrc-Hrp1 T3SS to inject effector proteins and thus subvert signalling pathways of their plant hosts. This secretion system (Hrc-Hrp1 T3SS) and its effector proteins are responsible for the development of the characteristic disease symptoms on susceptible plants and the triggering of the Hypersensitive Response (HR) in resistant plants $[26,49,50,52]$.

Comparative genomics of closely related isolates or species of pathogenic bacteria provides a powerful tool for rapid identification of genes involved in host specificity and virulence [53]. In this work, we reported sequence similarity searches, phylogeny analysis and prediction of the physicochemical characteristics of the hypothetical T3SS-2 proteins, as well as gene synteny analysis of the T3SS-2 gene cluster in $P$. syringae pv phaseolicola 1448a, P. syringae pv oryzae str. $1 \_6$ and $P$. syringae pv tabaci ATCC11528 in order to characterize this recently identified gene cluster. This analysis revealed that the T3SS-2 most closely resembles the T3SS of the Rhc-T3SS family. It further typifies a second discrete subfamily (subgroup II) within the Rhc-T3SS family in addition to the ones represented by the $R$. etli T3SS (subgroup III) and the known Rhizobium-T3SS (subgroup I). Usually, the presence of two T3SS gene clusters in the same genome is not the result of gene duplication inside the species but rather the result of independent horizontal gene transfers. This may reflect progressive coevolution of the plant patho/symbiosystem to either colonize various hosts or interact with the plant in different disease/symbiotic stages.

In our phylogenetic analysis proteins encoded in the T3SS-2 cluster of $P$. syringae strains are grouped together with the Rhizobium NGR234 T3SS-2. This finding suggests the possibility of an ancient acquisition from a common ancestor for Rhizobium NGR234 T3SS2 and the P. syringae T3SS-2. T3SSs of the Rhizobium family possesses a GC-content in same range (59-62\%), 
a value lower than the chromosome average. Since the GC content of T3SS-2 is almost the same as that of the genome of the $P$. syringae strains, it is difficult to characterize the second T3SS gene cluster as a genomic island based solely on this criterion. However, the genome sequencing of two other members of $P$. syringae [pathovars tomato DC3000, syringae B728A] revealed the total absence of a T3SS-2 like cluster.

The T3SS-2 gene cluster found in $P$. syringae pv phaseolicola 1448a, P. syringae pv oryzae str.1_6, P. syringae pv tabaci and of Rhizobium sp. NGR234, is also present in $P$. syringae pv aesculi (strains NCPPB 3681 and 2250)[54], P. syringae pv savastanoi (str. NCPPB 3335) [55], P. syringae pv glycinea (strains: B076 and race 4) [56], $P$. syringae pv lachrymans str. M301315 (GenBank: AEAF01000091.1), P. syringae pv actinidiae str. M302091 (GenBank: AEAL01000073.1), P. syringae pv. morsprunorum str. M302280PT (GenBank: AEAE01000259.1) and P. syringae Cit 7 (GenBank: AEAJ01000620.1). This T3SS-2 defines a distinct lineage in the Rhc T3SS family of at least the same evolutionary age as the split between the NGR234 T3SS-2 from the other rhizobial T3SSs.

In light of these findings, there are two plausible scenarios. One is that $P$. syringae acquired the T3SS-2 cluster from an ancient donor which is common both to $P$. syringae and the Rhizobium sp. NGR234 T3SS-2, before the diversification of the $P$. syringae pathovars from each other, followed by subsequent loss from certain members of the group. Another scenario is that multiple horizontal transfers from hypothetical donors into selected pathovars/strains occurred after their diversification. The present data set does not allow us to consider whether the hypothesis of an earlier acquisition followed by subsequent loss from members such as $P$. syringae pv tomato DC3000 might be considered more likely than several independent acquisitions.

The genes $h r c_{I I} N$ and $h r c_{I I} V$ in $P$. syringae pv tabaci and $P$. syringae pv oryzae T3SS-2 clusters were split into at least two open reading frames in various positions suggesting possibly that they might be degenerate pseudogenes, while the $h_{r c} C 2$ gene in $P$. syringae pv tabaci is further split in two ORFs as well (Figure 4). However, this is not the case for the $P$. syringae pv phaseolicola 1448a, $P$. syringae pv savastanoi and $P$. syringae pv aesculi T3SS2 where all these genes remain intact while $h r c_{I I} C 1$ and $h r c_{I I} N$ transcripts were observed in $P$. syringae pv phaseolicola 1448a T3SS-2 case (Figure 4). Remarkably, the T3SS-2 genes expression was even higher in rich compared to minimal medium (Figure 3). Minimal media of slightly acidic $\mathrm{pH}$ are thought to simulate in planta conditions and promote expression of the P. syringae T3SS-1 and effectors $[24,57,58]$. Such genes typically possess conserved motifs ( $h r p$ boxes) in their promoter regions and are transcriptionally controlled by the alternative sigma factor HrpL. However, the T3SS-2 operons in the P. syringae pv phaseolicola 1448a genome do not appear to have hrp boxes like those found in T3SS-1 genes of P. syringae strains [27]. This suggests that Psph 1448a does restrict T3SS-2 expression to in planta conditions and the potential contribution of the T3SS-2 in P. syringae life cycle may not be connected with the phytopathogenic potential of this species. Further functional studies are thus needed to reveal the exact biological roles of this secretion system in bacterium-plant interactions or other aspects of the bacterial life cycle. Suppression of other secretion systems under the T3SS-1 inducing conditions has also been reported for the T6SS of $P$. syringae pv syringae $\mathrm{B} 728 \mathrm{a}$ [59] as well as for the $P$. aeruginosa T3SS [60], which do not appear to play a role in plant pathogenesis $[59,61,62]$.

Gene transfer between phylogenetically remote bacteria would be favored by colonization of the same environmental niche [63]. In nature, Rhizobium is normally viewed as a microbe that survives saprophytically in soil, in nitrogen fixing nodules of legumes or as endophytes in gramineous plants, for example field grown [64] and wild rice [65]. $P$. syringae pv phaseolicola $1448 \mathrm{~A}$ and $P$. syringae $\mathrm{pv}$ oryzae str.1_6 are pathogens of the common bean and rice, respectively, while Rhizobium sp. NGR234 forms nitrogen fixing nodules with more legumes than any other microsymbiont [38]. Thus, there is ample opportunity for niche overlap between at least one of the P. syringae pathovars possessing T3SS-2 and Rhizobium sp. NGR234. At this point, a role for T3SS-2 in host-bacterium interactions for the rhizobia or the $P$. syringae strains possessing the system remains to be established and it is not obvious why these bacteria maintain a second T3SS gene cluster in their genome. Functional analysis and genome sequencing of more rhizobia that share common niches with $P$. syringae as well as the sequencing of more $P$. syringae pathovar genomes may shed light into these questions.

\section{Additional files}

\begin{abstract}
Additional file 1: Figure S1. Unrooted neighbor-joining phylogenetic tree of SctQ proteins of flagellar and non-flagellar T3S proteins. The tree was calculated by CLUSTALW (1.82) using bootstrapping (500 replicates) as a method for deriving confidence values for the groupings and was drawn by MEGA 4.0. Bootstrap values are indicated in each branching point. Scale bar represents numbers of substitution per site. The arrow indicates a possible position of root so that the tree will be compatible with the monophyly of the flagellar T3SS. Consistently with phylograms based on other conserved proteins of the Pph T3SS-2, the Hrc/lQ polypeptide does not fall into any of the two Hrc1/Hrc2 T3SS families but it is grouped with the Rhc family.
\end{abstract}

Additional file 2: Figure S2. Unrooted neighboring joining tree including all known SctV T3SS families and the flagelar proteins. Bootstrap values are percentages of 500 repetitions taking place. Multiple alignment performed with ClustalW.

Additional file 3: Figure S3. Evolutionary relationships of $250 \mathrm{HrCN}$ / YscN/Flil proteins. A. The phylogram of $253 \mathrm{SctN}$ sequences subdivided in 
seven main families, depicted with different colors and named according to [8], while the flagellum proteins are depicted in black. The evolutionary history was inferred as in case of Figure 2. B. The Rhc T3SS clade as derived from the phylogram in A, groups clearly the P. syringae Hrc, $V$ sequences close to the Rhc ${ }_{\text {IV }}$ protein of the Rhizobium sp. NGR234 T3SS-2. The values at the nodes are the bootstrap percentages out of 1000 replicates. The locus numbers or the protein accession number of each sequence is indicated.

Additional file 4: Table S1. Sequence comparisons of T3SS-2 proteins with proteins from from subgroups I-III of Rhc T3SS gene clusters. Percentage identities of various T3SS proteins in comparison to the Pph T3SS-2 proteins. Pph T3SS-2 cluster shares a higher degree of common genes with T3SS-2 of Rhizobium sp. NGR234 than with Rhc T3SS gene clusters of subgroup I or III. Shading in grayscale is according to percentage identity.

Additional file 5: Figure S4. Multiple alignements with ClustalW version 1.8 [19] for A) RhcC1 proteins (ref|YP 274720.1| Hrc//C1 [Pseudomonas syringae pv. phaseolicola 1448a], reflZP 04589253.1] HrC//C1 [Pseudomonas syringae pv. oryzae str. 1_6], ref|YP 002824487.1| Rhc/lC [Rhizobium sp. NGR234], ref|NP 444156.1| NolW [Rhizobium sp. NGR234], ref|NP 106861.1| NOLW [Mesorhizobium loti MAFF303099], ref|NP 768451.1| RhcC1 [Bradyrhizobium japonicum USDA 110] and B) RhcC2 proteins (ref|ZP 04589255.1|Hrp//C2 [Pseudomonas syringae pv. oryzae str. 1_6], ref|YP 002824481.1| Rhcl/C2 [Rhizobium sp. NGR234], ref|NP 106858.1] RhcC2 [Mesorhizobium loti MAFF303099], ref|NP 768482.1| RhcC2 [Bradyrhizobium japonicum USDA 110] and ref|NP 444146.1| Y4X] [Rhizobium sp. NGR234]. Visualization of the alignment was performed in http://www.bioinformatics.org/sms2/color_align_cons.html.

Additional file 6: Figure S5. Sequence analysis for HrpO-like proteins The analysis of PSPPH_2532 (Hrp//O) indicates that this hypothetical protein belongs to the HrpO/YscO/FliJ family of T3SS proteins [5,33]. The same is evident for the sequence annotated as RhcZ in the T3SS-2 of Rhizobium sp. NGR342. Residues predicted in a-helical conformation are indicated in yellow and unfolded regions in red. Green areas indicate ordered regions. Residues for which a high propensity for coiled-coil formation is predicted are indicated in blue rectangular. Here a-helix prediction was performed with PsiPRED, disordered prediction with FOLDINDEX and coiled coils prediction with COILS. Accession numbers or loci numbers are: AAC25065 (HrpO), P25613 (Flij), AAB72198 (YscO), PSPPH_2532 (Hrp//O), NGR_b22960 (RhcZ), NGR234_462 (Y4yJ).

Additional file 7: Table S2. Codon Usage Bias Table.

\section{Authors' contributions}

ADG performed research, helped draft the manuscript, analysed results and prepared figures. PFS, VEF, SNC and NM performed research, analysed results and critically appraised the manuscript. NJP and MK designed research, supervised work, organized financial support and critically appraised the manuscript. All authors read and approved the final manuscript.

\section{Acknowledgements}

We thank loanna Eleftheriadou for assistance in the initial search for T3SS related ORFs in the P. syringae pv phaseolicola 1448a genome. This work was supported by PENED, PYTHAGORAS and PEP (KP-15) grants from the Greek Ministry of Education, GSRT and the EPEAEK-Plant Molecular Biology and Biotechnology and the Protein Biotechnology graduate programs. S.N.C. was recipient of an Onassis Foundation fellowship and a GSRT post-doctoral grant. V.E.F is supported by a Marie Curie Reintegration Grant.

\section{Author details}

'Department of Biology, University of Crete, Vasilika Vouton, P.O. Box 2208, Heraklion, Crete GR 71409, Greece. ${ }^{2}$ Institute of Molecular Biology \& Biotechnology, Foundation of Research \& Technology, Vasilika Vouton Heraklion, Crete GR 71110, Greece. ${ }^{3}$ Institute Pasteur, 28 rue du Docteur Roux, Paris 75015, France. ${ }^{4}$ School of Medicine, University of Crete, Vasilika Vouton, Heraklion, Crete GR 71409, Greece. ${ }^{5}$ Department of Molecular Biology and Genetics, Democritus University of Thrace, Alexandroupolis GR 68100, Greece.
Received: 14 May 2012 Accepted: 21 August 2012

Published: 2 September 2012

\section{References}

1. Economou A, Christie PJ, Fernandez RC, Palmer T, Plano GV, Pugsley AP: Secretion by numbers: protein traffic in prokaryotes. Mol Microbiol 2006, 62(2):308-319.

2. Tseng T-T, Tyler BM, Setubal JC: Protein secretion systems in bacterial-host associations, and their description in the Gene Ontology. BMC Microbiol 2009, 9(Suppl 1):S2. doi:10.1186/1471-2180-1189-S1181-S1182.

3. Cascales E, Christie PJ: The versatile bacterial type IV secretion systems. Nat Rev Microbiol 2003, 1(2):137-149.

4. Cornelis GR: The type III secretion injectisome. Nat Rev Microbiol 2006, 4:811-825.

5. Gazi AD, Charova SN, Panopoulos NJ, Kokkinidis M: Coiled-coils in type III secretion systems: structural flexibility, disorder and biological implications. Cell Microbiol 2009, 11(5):719-729.

6. Tampakaki AP, Skandalis N, Gazi AD, Bastaki MN, Sarris PF, Charova SN, Kokkinidis M, Panopoulos NJ: Playing the "Harp": evolution of our understanding of hrp/hrc Genes. Annu Rev Phytopathol 2010, 17:347-370.

7. Tampakaki AP, Fadouloglou VE, Gazi AD, Panopoulos NJ, Kokkinidis M: Conserved features of type III secretion. Cell Microbiol 2004, 6(9):805-816.

8. Troisfontaines P, Cornelis GR: Type III secretion: more systems than you think. Physiol 2005, 20:326-339.

9. Gophna U, Ron EZ, Graur D: Bacterial type III secretion systems are ancient and evolved by multiple horizontal-transfer events. Gene 2003, 312:151-163.

10. Altschul SF, Madden TL, Schffer AA, Zhang J, Zhang Z, Miller W, Lipman DJ: Gapped BLAST and PSI-BLAST: a new generation of protein database search programs. Nucleic Acid Res 1997, 25(17):3389-3402.

11. Prilusky J, Felder CE, Zeev-Ben Mordehai T, Rydberg EH, Man O, Beckmann JS, Silman IJ, Prilusky J, Felder CE, Zeev-Ben Mordehai T, Rydberg EH, Man O, Beckmann JS, Silman IJLS: FoldIndex@: a simple tool to predict whether a given protein sequence is intrinsically unfolded. Bioinf 2005, 21:3435-3438.

12. Jones DT: Protein secondary structure prediction based on position-specific scoring matrices. J Mol Biol 1999, 292(2):195-202.

13. Handbook. Totowa, New Jersey: Humana Press; 2005.

14. Lupas A, Van Dyke M, Stock J: Predicting coiled coils from protein sequences. Science 1991, 252:1162-1164.

15. Fischetti VA, Landau GM, Schmidt JP, Sellers P: Identifying periodic occurences of a template with applications to protein structure. Inform Process Let 1993, 45:11-18.

16. Kelley LA, MacCallum RM, Sternberg MJE: Enhanced genome annotation with structural profiles in the program 3D-PSSM. J Mol Biol 2000, 299:499-500.

17. McGuffin LJ, Bryson K, Jones DT: The PSIPRED protein structure prediction server. Bioinfor 2000, 16:404-405.

18. Librado P, Rozas J: DnaSP v5: A software for comprehensive analysis of DNA polymorhism data. Bioinfor 2009, 25:1451-1452.

19. Thompson JD, Higgins DG, Gibson TJ: ClustalW: Improving the sensitivity of progressive multiple sequence alignment through sequence weighting, position, specific gap penalties, and weight matrix choice. Nucleic Acid Res 1994, 22:4673-4680.

20. Saitou N, Nei M: The neighbor-joining method: a new method for reconstructing phylogenetic trees. Mol Biol Evol 1987, 4(4):406-425.

21. Tamura K, Dudley J, Nei M, Kumar S: MEGA4: Molecular Evolutionary Genetics Analysis (MEGA) software version 4.0. Mol Biol Evol 2007, 24:1596-1599.

22. Felsenstein J: Confidence limits on phylogenies: an approach using the bootstrap. Evolution 1985, 39(4):783-791.

23. Zuckerkandl E, Pauling L: Evolutionary divergence and convergence in proteins. New York: Academic; 1965.

24. Rahme LG, Mindrinos MN, Panopoulos NJ: Plant and environmental sensory signals control the expression of hrp genes in Pseudomonas syringae pv. phaseolicola. J Bacteriol 1992, 174(11):3499-3507.

25. Rijpensa N, Jannesb G, Hermana L: Messenger RNA-based RT-PCR detection of viable Salmonella. Intern Diary J 2002, 12:233-238.

26. Hueck CJ: Type III protein secretion systems in bacterial pathogens of animals and plants. Microbiol Mol Biol Rev 1998, 62(2):379-433.

27. Xiao $Y$, Hutcheson SW: A single promoter sequence recognized by a newly identified alternate sigma factor directs expression of 
pathogenicity and host range determinants in Pseudomonas syringae. J Bacteriol 1994, 176(10):3089-3091.

28. Viprey V, Del Greco A, Golinowski W, Broughton WJ, Perret X: Symbiotic implications of type III protein secretion machinery in Rhizobium. Mol Microbiol 1998, 28(6):1381-1389.

29. Krause A, Doerfel A, Göttfert M: Mutational and Transcriptional Analysis of the Type III Secretion System of Bradyrhizobium japonicum. MPMI 2002, 15(12):1228-1235.

30. Kovács LG, Balatti PA, Krishnan HB, Pueppke SG: Transcriptional organisation and expression of nolXWBTUV, a locus that regulates cultivar-specific nodulation of soybean by Rhizobium fredii USDA257. Mol Microbiol 1995, 17:923-933.

31. Fadouloglou VE, Tampakaki AP, Glykos NM, Bastaki MN, Hadden JM, Phillips SE, Panopoulos NJ, Kokkinidis M: Structure of HrcQB-C, a concerved component of the bacterial type III secretion systems. Proc Natl Acad Sci USA 2004, 101:70-75.

32. Fadouloglou VE, Bastaki MN, Ashcroft AE, Phillips SEV, Panopoulos NJ, Glykos $N M$, Kokkinidis M: On the quaternary association of the type III secretion system HrcQB-C protein: experimental evidence differentiates among the various oligomerization models. J Struct Biol 2009, 166(2):214-225.

33. Gazi AD, Bastaki M, Charova SN, Gkougkoulia EA, Kapellios EA, Panopoulos NJ, Kokkinidis M: Evidence for a coiled-coil interaction mode of disordered proteins from bacterial type III secretion systems. J Biol Chem 2008, 283(49):34062-34068.

34. Pallen MJ, Beatson SA, Bailey CM: Bioinformatics analysis of the locus for enterocyte effacement provides novel insights into type-III secretion. BMC Microbiol 2005, 5:9.

35. Freiberg C, Fellay R, Bairoch A, Broughton WJ, Rosenthal A, Perret X: Molecular basis of symbiosis between Rhizobium and legumes. Nat 1997, 387:394-401.

36. Marie C, Broughton WJ, Deakin WJ: Rhizobium type III secretion systems: legume charmers or alarmers? Curr Opin Plant Biol 2001, 4(4):336-342.

37. González V, Bustos P, Ramírez-Romero MA, Medrano-Soto A, Salgado H, Hernández-González I, Hernández-Celis JC, Quintero V, Moreno-Hagelsieb G, Girard $L$, et al: The mosaic structure of the symbiotic plasmid of Rhizobium etli CFN42 and its relation to other symbiotic genome compartments. Gen Biol 2003, 4(6):R36.

38. Schmeisser C, Liesegang H, Krysciak D, Bakkou N, Le Quere A, Wollherr A Heinemeyer I, Morgenstern B, Pommerening-Roser A, Flores M, et al: Rhizobium sp. Strain NGR234 Possesses a Remarkable Number of Secretion Systems. Appl Environ Microbiol 2009, 75(12):4035-4045.

39. Petnicki-Ocwiega T, van Dijk K, Alfano JR: The hrpK operon of Pseudomonas syringae pv. tomato DC3000 encodes two proteins secreted by the type III (Hrp) protein secretion system: HopB1 and HrpK, a putative type III translocator. J Bacteriol 2005, 187:649-663.

40. Day JB, Plano GV: The Yersinia pestis YscY Protein Directly Binds YscX, a Secreted Component of the Type III Secretion Machinery. J Bacteriol 2000, 182(7):1834-1843

41. Duderstadt KE, Berger JM: AAA + ATPases in the Initiation of DNA Replication. Crit Rev Biochem Mol Biol 2008, 43:163-187.

42. Pallen MJ, Francis MS, Futterer $\mathrm{K}$ : Tetratricopeptide-like repeats in type-III-secretion chaperones and regulators. FEMS Microbiol Lett 2003, 223:53-60.

43. Darwin KH, Miller VL: Type III secretion chaperone-dependent regulation: activation of virulence genes by SicA and InvF in Salmonella typhimurium. EMBO J 2001, 20:1850-1862.

44. Joardar V, Lindeberg M, Jackson RW, Selengut J, Dodson R, Brinkac LM, Daugherty SC, DeBoy R, Durkin AS, Giglio MG, et al: Whole-Genome Sequence Analysis of Pseudomonas syringae pv. phaseolicola 1448A reveals divergence among pathovars in genes involved in virulence and transposition. J Bacteriol 2005, 187(18):6488-6498.

45. Sawada H, Suzuki F, Matsuda I, Saitou N: Phylogenetic analysis of Pseudomonas syringae pathovars suggests the horizontal gene transfer of $\operatorname{argK}$ and the evolutionary stability of hrp gene cluster. J Mol Evol 1999, 49:627-644.

46. Ettema TJG, Andersson SGE: The a-proteobacteria: the Darwin finches of the bacterial world. Biol Lett 2009, 5:391-393.

47. Fauvart M, Michiels J: Rhizobial secreted proteins as determinants of host specificity in the rhizobium-legume symbiosis. FEMS Microbiol Lett 2008, 285:1-9.
48. Beattie GA, Lindow SE: Bacterial colonization of leaves: a spectrum of Strategies. Phytopathol 1999, 89(5):353-359.

49. Grant SR, Fisher EJ, Chang JH, Mole BM, Dangl JH: Subterfuge and manipulation: Type III effector proteins of phytopathogenic bacteria. Annu Rev Microbiol 2006, 60:425-449.

50. He SY: Type III protein secretion systems in plant and animal pathogenic bacteria. Annu Rev Phytopathol 1998, 36:363-392.

51. Hirano SS, Upper CD: Bacteria in the Leaf Ecosystem with Emphasis on Pseudomonas syringae-a Pathogen, Ice Nucleus, and Epiphyte. Microbiol Mol Biol Rev 2000, 64:624-653.

52. Lindeberg M, Myers CR, Collmer A, Schneider DJ: Roadmap to new virulence determinants in Pseudomonas syringae: Insights from comparative genomics and genome organization. Mol Plant Microbiol Inter 2008, 21:685-700

53. da Silva AC, Ferro JA, Reinach FC, Farah CS, Furlan LR, Quaggio RB, Monteiro-Vitorello CB, Van Sluys MA, Almeida NF, Alves LM, et al: Comparison of the genomes of two Xanthomonas pathogens with differing host specificities. Nature 2002, 417:459-463.

54. Green S, Studholme DJ, Laue BE, Dorati F, Lovell H, Arnold D, Cottrell JE, Bridgett S, Blaxter M, Huitema E, et al: Comparative genome analysis provides insights into the evolution and adaptation of Pseudomonas syringae pv. aesculi on Aesculus hippocastanum. PLoS One 2010, 5(4): e10224.

55. Rodríguez-Palenzuela P, Matas IM, Murillo J, López-Solanilla E, Bardaji L, Pérez-Martínez I, Rodríguez-Moskera ME, Penyalver R, López MM, Quesada J, et al: Annotation and overview of the Pseudomonas savastanoi pv. savastanoi NCPPB 3335 draft genome reveals the virulence gene complement of a tumour-inducing pathogen of woody hosts. Environ Microbiol 2010, 12(6):1604-1620.

56. Qi M, Wang D, Bradley CA, Zhao Y: Genome sequence analyses of Pseudomonas savastanoi pv. glycinea and subtractive hybridization-based comparative genomics with nine pseudomonads. PLoS One 2011, 6(1):e16451.

57. Huynh TV, Dahlbeck D, Staskawicz BJ: Bacterial blight of soybean: regulation of a pathogen gene determining host cultivar specificity. Science 1989, 245(4924):1374-1377.

58. Clarke CR, Cai R, Studholme DJ, Guttman DS, Vinatzer BA: Pseudomonas syringae strains naturally lacking the classical P. syringae hrp/hrc Locus are common leaf colonizers equipped with an atypical type III secretion system. Mol Plant Microbe Interact 2010, 23(2):198-210.

59. Records AR, Gross DC: Sensor kinases RetS and LadS regulate Pseudomonas syringae type VI secretion and virulence factors. J Bacteriol 2010, 192(14):3584-3596.

60. Mougous JD, Gifford CA, Ramsdell TL, Mekalanos JJ: Threonine phosphorylation post-translationally regulates protein secretion in Pseudomonas aeruginosa. Nat Cell Biol 2007, 9(7):797-803.

61. Lesic B, Starkey M, He J, Hazan R, Rahme LG: Quorum sensing differentially regulates Pseudomonas aeruginosa type VI secretion locus I and homologous loci II and III, which are required for pathogenesis. Microbiology 2009, 155(Pt 9):2845-2855.

62. He J, Baldini RL, Deziel E, Saucier M, Zhang Q, Liberati NT, Lee D, Urbach J, Goodman HM, Rahme LG: The broad host range pathogen Pseudomonas aeruginosa strain PA14 carries two pathogenicity islands harboring plant and animal virulence genes. Proc Natl Acad Sci USA 2004, 101:2530-2535.

63. Fall S, Mercier A, Bertolla F, Calteau A, Gueguen L, Perrii G, Vogel TM, Simonet P: Horizontal Gene Transfer Regulation in Bacteria as a $\beta €$ Spandrel $\beta €$ of DNA Repair Mechanisms. PLoS One 2007, 2(10):e1055.

64. Youssef YG, Rizk RY, Corich V, Squartini A, Ninke K, Philip-Hollingsworth S, Orgambide G, De Bruijn F, Stoltzfus J, Buckley D, et al: Natural endophytic association between Rhizobium leguminosarum bv. trifolii and rice roots and assessment of its potential to promote rice growth. Plant Soil 1997, 194:99-114.

65. Peng G, Yuan Q, Li H, Zhang W, Tan Z: Rhizobium oryzae sp. nov., isolated from the wild rice Oryza alta. Int J Syst Evol Microbiol 2008, 58:2158-2163.

doi:10.1186/1471-2180-12-188

Cite this article as: Gazi et al:: Phylogenetic analysis of a gene cluster encoding an additional, rhizobial-like type III secretion system that is narrowly distributed among Pseudomonas syringae strains. BMC Microbiology 2012 12:188. 